\title{
Monte Carlo study of the operation of GaN planar nanodiodes as sub-THz emitters in resonant circuits
}

\author{
B G Vasallo ${ }^{1}$, J F Millithaler ${ }^{1}$, I Íñiguez-de-la-Torre ${ }^{1}$, T González ${ }^{1}$, \\ G Ducournau $^{2}$, C Gaquière ${ }^{2}$ and $J$ Mateos $^{1}$ \\ ${ }^{1}$ Departamento de Física Aplicada, Universidad de Salamanca, Plaza de la Merced s/n, 37008 Salamanca, \\ Spain \\ ${ }^{2}$ IEMN/Université de Lille 1, UMR CNRS 8520, Avenue Poincaré, 59650 Villeneuve d'Ascq, France \\ E-mail: bgvasallo@usal.es
}

Received 9 April 2014, revised 9 September 2014

Accepted for publication 15 September 2014

Published 17 October 2014

\begin{abstract}
A study of the high-frequency performance of GaN-based asymmetric self-switching diodes (SSDs) designed for a room-temperature sub-THz Gunn emission, and connected to a resonant RLC parallel circuit, is reported. With the aim of facilitating the achievement and control of Gunn oscillations, which can potentially allow the emission of $\mathrm{THz}$ radiation by GaN SSDs, a time-domain Monte Carlo (MC) theoretical study is provided. The simulator has been validated by comparison with the $I-V$ curves of similar fabricated structures, including the possibility of heating effects. A V-shaped SSD has been found to be more efficient than the square one in terms of the DC to AC conversion efficiency $\eta$. Indeed, according to our MC results, a value of $\eta$ of at least $0.35 \%$ @ $270 \mathrm{GHz}$ can be achieved for the V-shaped SSD at room temperature by using an adequate resonant circuit. This value can be increased up to $0.80 \%$, even when considering the heating effects, with appropriate RLC elements. Furthermore, simulations show that when several diodes are fabricated in parallel in order to enhance the emitted power, there is no synchronization between the oscillations of all the SSDs; however, the phase-shift effects can be solved using a synchronized current injection by the attachment of a resonant circuit.
\end{abstract}

Keywords: self-switching diode, GaN, Monte Carlo simulations, Gunn oscillations, resonant circuits

(Some figures may appear in colour only in the online journal)

\section{Introduction}

Strong interest has been shown in investigating the $\mathrm{THz}$ frequency range due to the countless applications in a wide range of fields [1]. In this context, planar self-switching diodes (SSDs), originally proposed by A. M. Song et al [2], are being explored with the aim of achieving $\mathrm{THz}$ emission, since their topology is especially appropriate for the development of Gunn oscillations [3]. This device provides an interesting non-linear behavior (based on the presence of surface charges and a lateral field effect) and can be fabricated with a simple single-step lithographic process. The operation of SSDs as detectors can approach the $\mathrm{THz}$ range at room temperature [4], as experimentally demonstrated in references
[5-7]. On the emitter side, in order to achieve the highest frequencies and emitted power, promising materials such as GaN have been recently explored [8-11] due to its very high breakdown field and saturation velocity and the possibility of high temperature operation. In fact, in recent years much effort has been dedicated to the fabrication of vertical GaN Gunn diodes [12], so far without success. The planar design of SSDs allows for the enhancement of power emission by means of parallelization, which also provides more flexibility in the design. For example, thanks to the possibility of introducing several devices with different characteristic frequencies, a better broadband performance can be achieved.

In order to experimentally achieve and control the oscillating behavior, Gunn diodes, acting as free oscillators, 


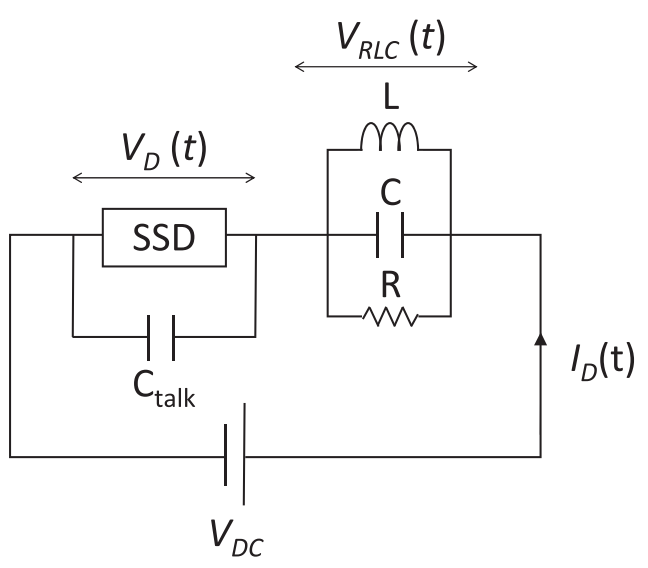

Figure 1. Circuit configuration of the series connection of the SSD with the parallel RLC tank.

have to be embedded in a resonant circuit to be operated, which can be a parallel RLC circuit connected in series with the devices [11-13]. Up to the present time there has not been any experimental evidence of Gunn oscillations in GaN-based devices, in part because the most likely solution for overcoming the practical problems, which is the fabrication of diodes with integrated resonators (quite complicated because of the technological difficulties to achieve resonant circuits at targeted oscillating frequencies), has not been possible yet [14]. In order to advance in such a direction, physical models that include the intrinsic physical behavior of the SSDs and their coupling to a resonant circuit must be used. In particular, particle-simulation time-domain methods, such as the Monte Carlo (MC) [15] model, are highly appropriate for the analysis of Gunn oscillations [8, 10, 11], but they are difficult to apply for circuit simulation (where a frequency-domain analysis is more efficient). Other issues to be considered when dealing with the Gunn effect in GaN diodes are heating effects [12]. As explained in [16], when the diodes operate under only a DC voltage, the use of a V-shaped design for the channel, instead of the standard square one, allows a decrease in the threshold voltage needed for the onset of Gunn oscillations, which are achieved with a much lower DC current, which helps to alleviate the thermal problems.

In this context, the aim of this work is to analyze the time-domain operation of both V-shaped (VS-SSD) and square (SQ-SSD) GaN planar Gunn SSDs when connected to a resonant-circuit by coupling a semi-classical ensemble MC model, employed for the simulation of the intrinsic device, with a consistent solution of a parallel RLC circuit connected in series [17] to the intrinsic device, as shown in figure 1. Heating effects are also implemented in the model. The two types of designs, SQ- and VS-SSDs, are analyzed and compared in terms of oscillation frequencies, DC to AC conversion efficiency, and thermal performance. The use of a resonant circuit to achieve the synchronization of the Gunn oscillations taking place in individual channels of an array of parallel SSDs is also illustrated. Finally, the effect of the parasitic elements that may affect the practical implementation of the oscillators, mainly the crosstalk capacitance $\mathrm{C}_{\text {talk }}$,
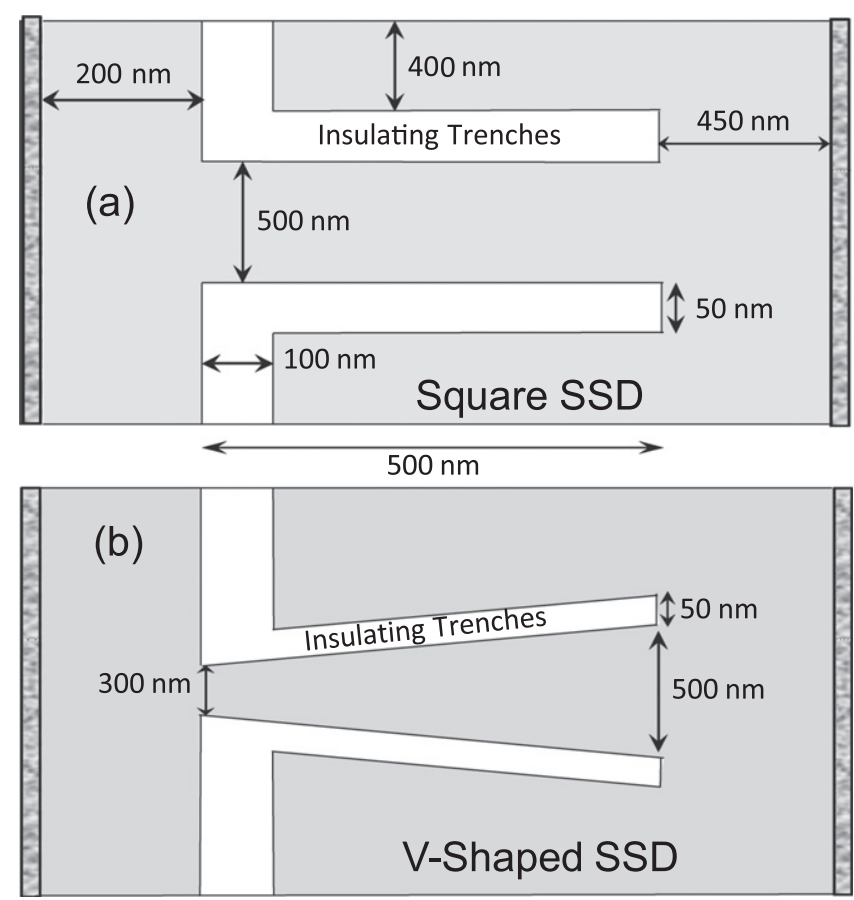

Figure 2. Sketch of the geometry of the GaN (a) SQ-SSD and (b) VS-SSD under analysis.

as a function of the number of SSDs to be put in parallel are discussed.

The present paper is organized as follows. In section 2 the devices under analysis and the MC model employed, whose validity is verified by comparison with experimental $I-$ $V$ curves, are described. The study of the operation of the oscillators is presented in section 3, and afterwards, in section 4 , the most important conclusions of this work are drawn.

\section{SSDs under study and physical models}

The schematic topology of the GaN planar (a) SQ-SSDs and (b) VS-SSDs under analysis are shown in figure 2, both providing similar current levels (see figure 3). The simulated devices replicate the topology of real devices fabricated on an epitaxial layer consisting of an $\mathrm{Al}_{0.3} \mathrm{Ga}_{0.7} \mathrm{~N} / \mathrm{GaN}(1.8 \mu \mathrm{m})$ heterojunction (Ga-face) grown by metal oxide chemical vapor deposition on a high resistivity silicon (111) substrate. The two-dimensional electron gas in the quantum well was $23 \mathrm{~nm}$ below the surface covered by $5 \mathrm{~nm}$ of $\mathrm{S}_{\mathrm{i}} \mathrm{O}_{2}$ for passivation.

\subsection{Monte Carlo model}

For the calculations we make use of a semiclassical ensemble MC simulator self-consistently coupled with a two-dimensional (2D) Poisson solver, whose validity has been previously checked $[4,8,18]$ and has been very useful for the understanding of the physical behavior of SSDs and the optimization of their performances (mainly as detectors). Electron transport in $\mathrm{GaN}$ is modeled by three non-parabolic 


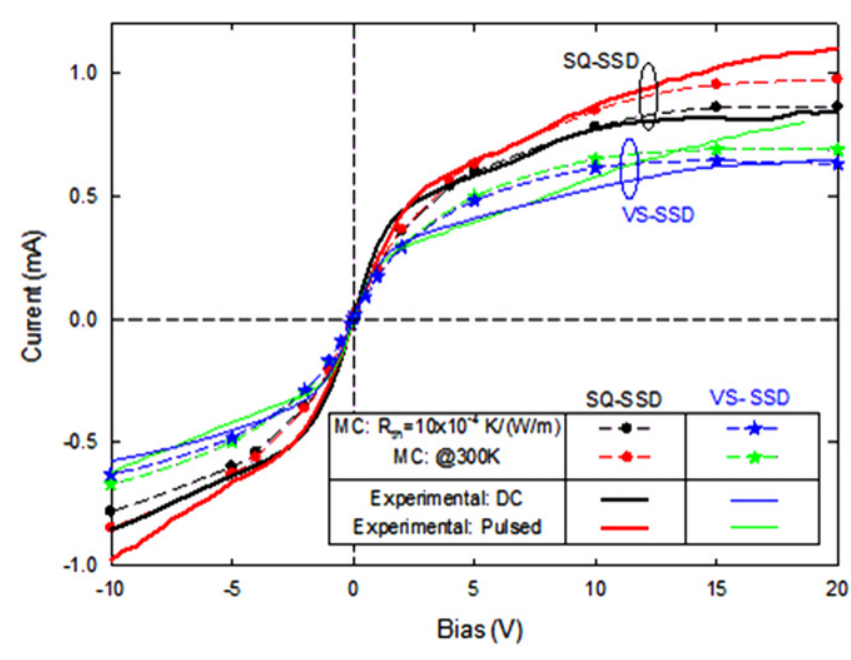

Figure 3. Experimental and MC $I-V$ characteristics for the GaN SQand VS-SSD with the geometries sketched in figure 2. Simulations are performed both at $300 \mathrm{~K}$ and considering $R_{\mathrm{th}}=10 \times 10^{-4} \mathrm{~K} /$ $(\mathrm{Wm})$, in order to reproduce the experimental $I-V$ curves measured in pulsed conditions and in DC, respectively.

spherical valleys $\left(\Gamma_{1}, \mathrm{U}\right.$, and $\left.\Gamma_{3}\right)$ with intervalley, acoustic, and optical phonons, ionized impurities, and piezoelectric scatterings [19]. To model the geometry of SSDs based on the $\mathrm{AlGaN} / \mathrm{GaN}$ heterojunction, the so-called top view simulations [4, 8, 18, 20-23] have been employed. The real layer structure of the devices is not included (just the GaN channel), while two ad hoc parameters must be considered, namely: (i) a virtual background net doping $N_{\mathrm{Db}}$, which is assigned to the simulated $\mathrm{GaN}$ layer (although the associated scattering is not considered) in order to account for the fixed charges at the top surface and the heterojunction; and (ii) a negative surface charge density $\sigma$, which is assigned to the semiconductor-air interfaces to account for the influence of the surface states originated during the etching process. For both devices SQ- and VS-SSDs, a value of $N_{\mathrm{Db}}=5 \times 10^{17} \mathrm{~cm}^{-3}$ has been used, while $\sigma$ is updated consistently with the surrounding electron concentration in order to reproduce the correct behavior of the device [21]. The simulation domain is discretized in meshes of $10 \mathrm{~nm} \times 10 \mathrm{~nm}$ (finite differences solution of the Poisson equation is done), small enough for a correct description of the leaning interfaces of the VS-SSD. The non-simulated dimension used to calculate the absolute values of the current [4] has been considered to be $\mathrm{Z}=n_{\mathrm{s}} / N_{\mathrm{Db}}=0.1 \mu \mathrm{m}$ (taking as a base the experimental value of the electron sheet concentration, $n_{\mathrm{s}}=5 \times 10^{12} \mathrm{~cm}^{-2}$ ).

\subsection{Thermal effects}

In order to account for the thermal effects in the MC simulations, a self-consistent method making use of the thermal resistance of the structure has been implemented [24]. Initially, the average output current is evaluated over a certain period $I_{\mathrm{MC}}$, and the lattice temperature $T_{\text {latt }}$ is updated according to the expression $T_{\text {latt }}=300 \mathrm{~K}+R_{\mathrm{th}} I_{\mathrm{MC}} V$, where
$R_{\mathrm{th}}$ is the thermal resistance of the device and $V$ the bias voltage. The lattice temperature, and consequently the contact injection and scattering rates, are thus iteratively adapted selfconsistently to the power dissipated within the device.

In order to characterize the thermal effects appearing in our devices, in figure 3 the MC $I-V$ curves calculated (i) at room temperature and ii) using a thermal resistance of $R_{\mathrm{th}}=10 \times 10^{-4} \mathrm{~K} /(\mathrm{W} / \mathrm{m})$ are compared to the experimental $\mathrm{DC}$ and pulsed characteristics. The setup used to perform the experimental measurements on the SSDs consists of a probe station connected to a semiconductor analyzer Keithley 4200 SCS with a pulse measurement unit (Keithley 4225-PMU) and a remote amplifier/ switch (Keithley 4225-RPM), which allows us to perform ultrafast pulsed $I-V$ measurements. We have used a pulse width of $770 \mathrm{~ns}$ with a period of $0.1 \mathrm{~ms}$, which provides a very short duty cycle of less than $1 \%$. Figure 3 shows that the low bias region is similar for DC and pulsed measurements, but for $V>10 \mathrm{~V}$, when the dissipated power becomes significant, there are clear differences: the current decreases as the heating is intensified by the higher bias. A good agreement is found between the pulsed (heatingless) characteristic and the MC simulation at room temperature. For the DC case, several values for $R_{\mathrm{th}}$ were tested in the thermal MC simulations, obtaining the best agreement with the experiments done in the SQ-SSD when a value of $R_{\mathrm{th}}=10 \times 10^{-4} \mathrm{~K} /(\mathrm{W} / \mathrm{m})$ is used (not far from the thermal resistance found for GaN HEMTs [25, 26]). Under these conditions the simulations show that the devices reach a maximum temperature of about $500 \mathrm{~K}$ at $20 \mathrm{~V}$, also in good agreement with the experimental findings. MC simulations of Gunn oscillations in GaN SSDs including thermal effects show that the heating leads to a decrease of the oscillation frequency due to a lower drift velocity of the Gunn domain. However, even if the temperature increase can be as high as $200 \mathrm{~K}$, the heating is not enough to kill the Gunn oscillations [16-24].

\subsection{Resonant circuit}

In order to represent the influence of a resonant circuit on the oscillation capability of the diodes, the intrinsic MC simulations of the diodes have been coupled to the resolution of a parallel RLC resonant circuit connected in series with the diode [17]. The circuit configuration is sketched in figure 1. The circuit equations to be solved are the following:

$$
V_{\mathrm{DC}}=V_{\mathrm{D}}+V_{\mathrm{RLC}}
$$

where $V_{\mathrm{D}}$ and $V_{\mathrm{RLC}}$ are the voltage drops at the terminals of the diode and the RLC circuit, respectively. The total current flowing through the $\mathrm{SSD}, j_{\mathrm{D}}$, is:

$$
j_{\mathrm{D}}=C d V_{\mathrm{RLC}} / d t+j_{L}+V_{\mathrm{RLC}} / R,
$$

with $j_{L}$ being the current flowing through the inductance $L$, which can be obtained through:

$$
V_{\mathrm{RLC}}=L d j_{L} / d t \text {. }
$$



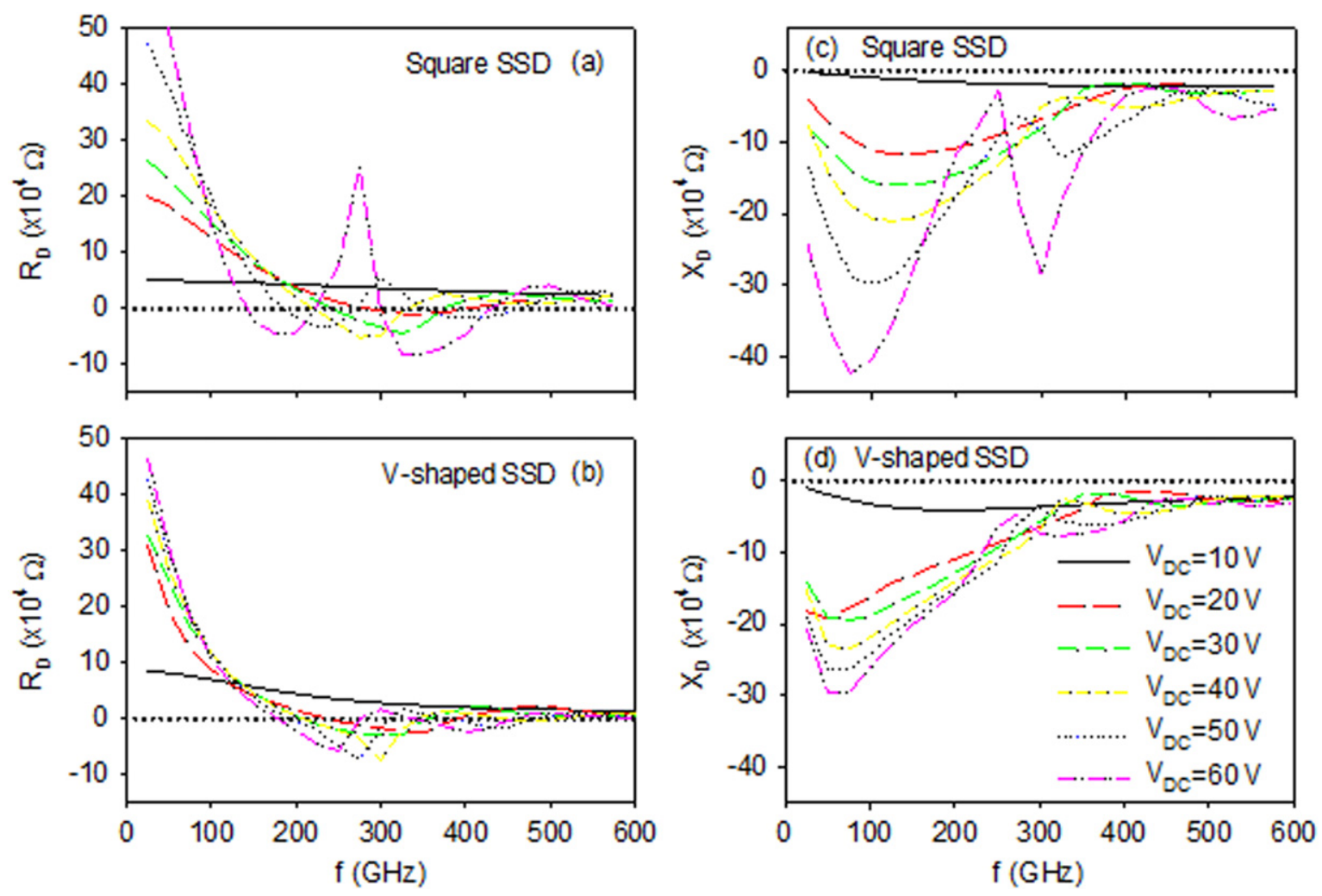

Figure 4. (a), (b) Real, $R_{\mathrm{D}}$, and (c), (d) imaginary part, $X_{\mathrm{D}}$, of the impedance at $300 \mathrm{~K}$ versus frequency for (a), (c) the SQ-SSD and (b), (d) the VS-SSD sketched in figures 2(a) and (b), respectively, at room temperature.

On the other hand, the total current flowing through the diode has two components:

$$
j_{\mathrm{D}}=j_{v}+C_{\mathrm{D}} d V_{\mathrm{D}} / d t
$$

The drift component of the current $j_{v}$ is directly calculated by the MC technique from the particle velocities, following the generalized Ramo-Shockley theorem [27], and the displacement current $j_{\text {dis }}=C_{\mathrm{D}} d V_{\mathrm{D}} / d t$ is due to the time-varying potentials at the electrodes of the diode, $C_{\mathrm{D}}$ being its capacitance. The parasitic effect of the crosstalk capacitance $\left(\mathrm{C}_{\text {talk }}\right.$, not included in the MC model) can be easily accounted for by adding its value to $C_{\mathrm{D}}$. The coupling of the circuit equations with the MC simulator is carried out following a finite-difference time-domain scheme, using the same time step as the simulator. The voltage drop at the terminals of the diode $V_{\mathrm{D}}$, used to solve the Poisson equation, is updated at each time step, with the solution of the previous system of equations obtained with the value of $j_{v}$ provided by the MC simulation [28].

The results of $\mathrm{MC}$ simulations considering one single diode (using a resonant circuit with $\mathrm{R}_{1}, \mathrm{~L}_{1}$, and $\mathrm{C}_{1}$ ) can be extrapolated to the case of the parallelization of N SSDs (in order to decrease their high impedance and increase the delivered power), as long as a resonant circuit having $\mathrm{R}_{\mathrm{N}}=\mathrm{R}_{1} / \mathrm{N}, \mathrm{L}_{\mathrm{N}}=\mathrm{L}_{1} / \mathrm{N}$ and $\mathrm{C}_{\mathrm{N}}=\mathrm{C}_{1} \times \mathrm{N}$ is used.

Finally, in order to analyze the diodes' capability as emitters, the dissipated DC power $\left(P_{\mathrm{DC}}\right)$ and the time-average AC power $\left(P_{\mathrm{AC}}\right)$ are evaluated, and the conversion efficiency is calculated as

$$
\eta=-P_{A C} / P_{\mathrm{DC}}
$$

Thus, negative values of $\eta$ indicate a resistive behavior of the diode, while positive values mean $\mathrm{AC}$ generation from the DC bias [29].

\section{Results}

\subsection{Generation bands}

Firstly, the impedance (both real $R_{\mathrm{D}}$ and imaginary $X_{\mathrm{D}}$ parts) of the SQ- and VS-SSDs shown in figure 2 has been estimated by the application of a sinusoidal voltage $V(t)=V_{\mathrm{DC}}+V_{\mathrm{AC}} \cos (2 \pi f t)$ with $V_{\mathrm{AC}}=1 \mathrm{~V}$ [29]. Figure 4 presents $R_{\mathrm{D}}$ and $X_{\mathrm{D}}$ as a function of frequency for several values of $V_{\mathrm{DC}}$. Heating effects are not initially considered. For both SQ- and VS-SSDs, negative values of the differential resistance appear at certain frequencies, indicating latent Gunn self-oscillations in that frequency range. In the SQ$\mathrm{SSD}$, the negative differential resistance appears between $250-380 \mathrm{GHz}$ at $V_{\mathrm{DC}}=30 \mathrm{~V}$. With the direct analysis of the time-varying current, we have confirmed that self-oscillations appear with a frequency around $385 \mathrm{GHz}$ for $V_{\mathrm{DC}}=30 \mathrm{~V}$ when a DC voltage only is applied (without any resonant circuit). This is consistent with the fact that self-oscillations in Gunn diodes appear around the upper limit of the negative resistance frequency range, when the resistance tends to 


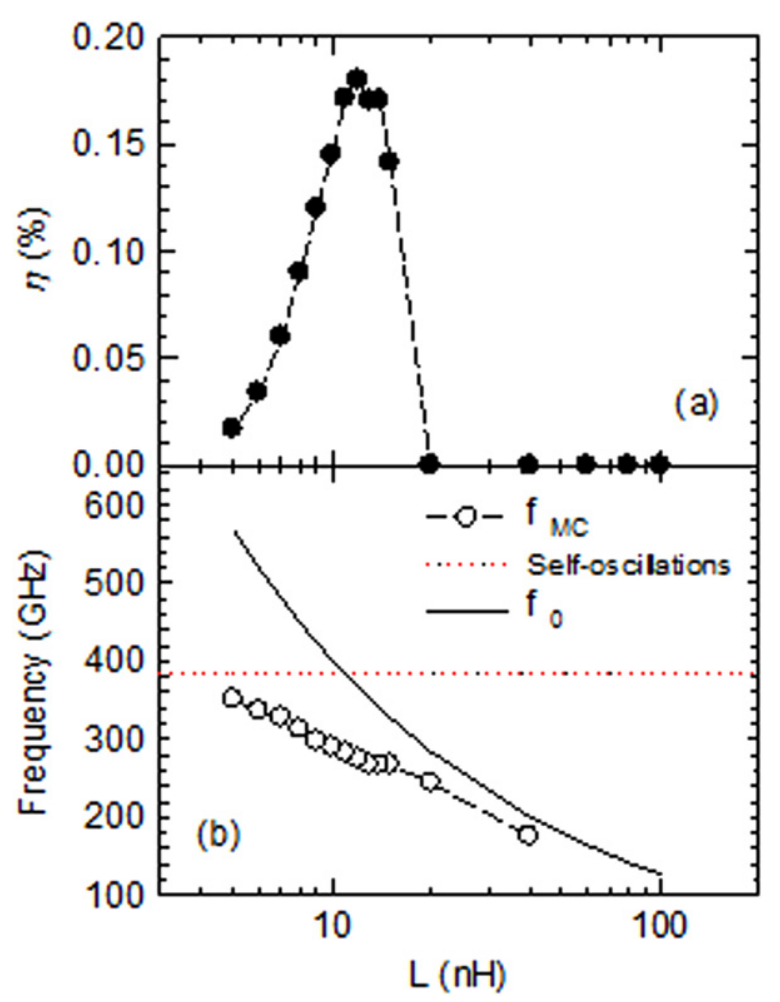

Figure 5. (a) DC to AC conversion efficiency $\eta$; and (b) theoretical resonant frequency of the circuit $f_{0}$ and frequency of the oscillations obtained with MC simulations $f_{\mathrm{MC}}$ in the SQ-SSD sketched in figure 2(a) connected in series with a resonant circuit, with $\mathrm{R}=0.5 \mathrm{M} \Omega, \mathrm{C}=0.01 \mathrm{fF}$, and $\mathrm{L}$ varying from $5-100 \mathrm{nH}$, at room temperature. The DC bias is $V_{\mathrm{DC}}=30 \mathrm{~V}$. The frequency corresponding to the self-oscillations found with only a DC bias applied (without the RLC tank) is indicated by the dotted line.

recover the zero value [30]. For the VS-SSD, the negative values of the resistance appear at lower applied voltages but with similar values than for the square diode. For the highest analyzed values of $V_{\mathrm{DC}}$, a second range of frequencies providing negative resistance appears $(150-220 \mathrm{GHz}$ and $300-420 \mathrm{GHz}$ at $V_{\mathrm{DC}}=60 \mathrm{~V}$ for the SQ-SSD), indicating the fact that current oscillations with two Gunn domains are also possible [8]. The imaginary part $X_{\mathrm{D}}$ is always negative, indicating a capacitive character that should be compensated by the external resonant circuit in order to implement an oscillator at a given frequency.

\subsection{Efficiency and frequency of oscillations}

To achieve Gunn oscillations, an RLC parallel circuit has been placed in series with a single diode. Figure 5 presents (a) the efficiency $\eta$; and (b) the theoretical expected frequency of the current oscillations $\left(f_{0}=1 /\left[2 \pi \sqrt{L\left(C+C_{\mathrm{D}}\right)}\right]\right)$ and the one obtained by MC simulations $\left(f_{\mathrm{MC}}\right)$, when considering a resonant circuit with $\mathrm{R}=0.5 \mathrm{M} \Omega, \mathrm{C}=0.01 \mathrm{fF}$, and $\mathrm{L}$ varying from $5-100 \mathrm{nH}$. The diode intrinsic geometric capacitance is $C_{\mathrm{D}}=0.0057 \mathrm{fF}$. As observed, the negative differential resistance of the SSD, combined with the feedback of the resonant circuit, leads to oscillations at a frequency lower than the characteristic resonant frequency of the RLC circuit $\left(f_{0}\right)$ and, as well, lower than that of Gunn self-oscillations (around $385 \mathrm{GHz}$ ). A maximum efficiency of $0.18 \%$ has been found for $\mathrm{R}=0.5 \mathrm{M} \Omega, \mathrm{C}=0.01 \mathrm{fF}$, and $\mathrm{L}=12 \mathrm{nH}$ (providing oscillations at $275 \mathrm{GHz}$ ). However, we have to stress that the time consuming $\mathrm{MC}$ simulations (computation time being around $4 \mathrm{~h}$ per point) and the required test-and-error procedure for the efficiency comparison in a post-processing stage allow us to check just a limited set of combinations of $\mathrm{R}, \mathrm{L}$, and $\mathrm{C}$ values. Thus, the efficiency could be further improved by optimizing these elements as well as the circuit configuration. The values adopted for the RLC resonant circuit may appear unfeasibly low, especially C. However, as will be discussed in Section 3.4, this problem can be solved by considering an array of $\mathrm{N}$ channels in parallel instead of a single SSD, with the corresponding scaling of the RLC values.

The achieved conversion efficiencies are consistent with other theoretical predictions in submicrometer GaN Gunn diodes [31]. Experimental results available for a mature technology like InP diodes offer conversion efficiencies around $1-3 \%$ in D-band [32] and $0.33 \%$ @ $280 \mathrm{GHz}$ [33]. Figure 5 also shows that a large frequency tunability (from 270-350 GHz) can be achieved at the expense of lower efficiencies. Even if these low values for the DC to AC conversion efficiency could be already experimentally useful, there is still much room for improvement by further optimizing the circuit design (with optimum values for $\mathrm{R}, \mathrm{L}$, and $\mathrm{C}$, and different circuit configurations) and the intrinsic characteristics of the SSD.

In order to establish a comparison between the SQ- and VS-SSDs, the dynamic behavior of both SSDs, coupled with the same resonant circuit with $\mathrm{R}=0.5 \mathrm{M} \Omega, \mathrm{C}=0.01 \mathrm{fF}$, and $\mathrm{L}=12 \mathrm{nH}$, has been analyzed for different DC biasing conditions. Figure 6 shows that the VS-SSD exhibits a threshold voltage for the onset of oscillations of $V_{\mathrm{DC}}=20 \mathrm{~V}$, lower than in the SQ-SSD, which needs $V_{\mathrm{DC}}>25 \mathrm{~V}$. The oscillation frequencies are in the range $265-275 \mathrm{GHz}$ in the VS-SSD, only slightly dependent on $V_{\mathrm{DC}}$ and with lower values than for the SQ-SSD (which provides 275-290 GHz). For both kinds of diodes, the efficiency presents a maximum value at $V_{\mathrm{DC}}=30 \mathrm{~V}$, which is significantly enhanced for the VS-SSD, reaching around $0.35 \%$. Nevertheless, we must remark again that, since just a limited set of R, L, and C has been analyzed, the exact values of the maximum efficiency obtained for the different geometries cannot be taken as definitive, but only as a reference for a possible experimental implementation of Gunn oscillators based on SSDs that should achieve at least those figures.

For $V_{\mathrm{DC}}>55 \mathrm{~V}$, two Gunn domains are built in the SQSSD (see figure 4), improving both the efficiency and the frequency of the oscillations, with the drawback that the dissipated DC power is increased $(60 \mathrm{~mW}$ per SSD at $\left.V_{\mathrm{DC}}=60 \mathrm{~V}\right)$. To further illustrate this point, the time-dependent values of the current and voltage in the circuits with SQand VS-SSDs at $V_{\mathrm{DC}}=30 \mathrm{~V}$ are shown in figures 7 (a) and (b), both exhibiting quite well-defined sinusoidal shapes at frequencies of about $275 \mathrm{GHz}$ and $260 \mathrm{GHz}$, respectively. The phase shift between voltage and current is slightly larger than a quarter of a period, thus producing the ellipse-like contours 


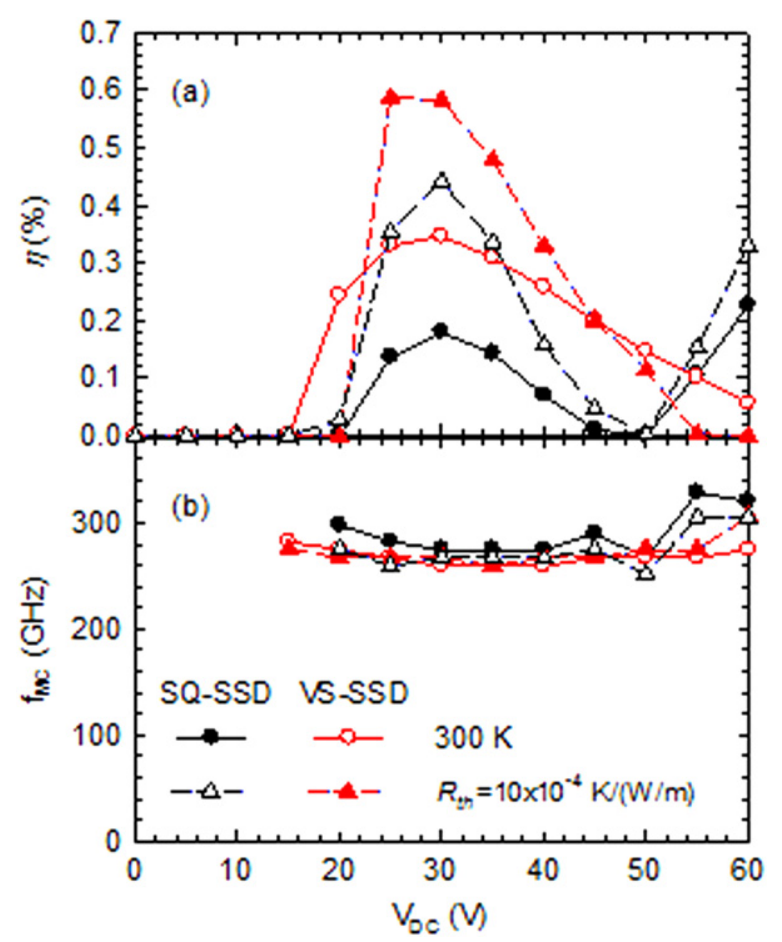

Figure 6. (a) DC to AC conversion efficiency $\eta$, and (b) frequency of the oscillations $f_{\mathrm{MC}}$ as a function of the applied DC voltage obtained with the MC simulations of the SQ- and VS-SSD sketched in figure 2(b), connected in series with an RLC tank with $R=0.5 \mathrm{M} \Omega$, $\mathrm{C}=0.01 \mathrm{fF}$, and $\mathrm{L}=12 \mathrm{nH}$. For both devices, the results at room temperature are compared with those obtained with the selfconsistent thermal MC model considering $R_{\mathrm{th}}=10 \times 10^{-4} \mathrm{~K} /(\mathrm{W} / \mathrm{m})$.

shown in the phase plane portrait of $\Delta I(t)-\Delta V(t)$ shown in figure 7(c). The shape of the contours of figure 7(c) allows us a better understanding of the values obtained for the efficiency $\eta$, since the area within the 2 nd and 4 th quadrants has a positive contribution to $\eta$ (current and voltage excursions have a different sign so that $P_{\mathrm{AC}}(t)$ is negative, and an AC power is generated), while, on the contrary, 1st and 3rd quadrants mean that the $\mathrm{AC}$ power is dissipated, thus having a negative contribution to $\eta$. The bias dependence of $\eta$ in the SQ-SSD can be thus explained. The decay when increasing the voltage from $V=30 \mathrm{~V}$ to a practically null value for $V=50 \mathrm{~V}$ is a result of the progressive reduction of the amplitude of the oscillations, while the subsequent increase for $V=60 \mathrm{~V}$ is mainly due to enhanced voltage variations. When comparing the SQ- and VS-SSDs at $30 \mathrm{~V}$, the enhanced efficiency of the latter comes not only from the larger current and voltage excursions but also from an increased contribution of the 2nd quadrant (negative AC voltage and positive AC current).

\subsection{Influence of heating}

Because of the parallelization needed to improve the generated power, the effect of the self-heating on the oscillations can be also of key importance. It is thus necessary to perform MC simulations that consider both the self-consistent temperature model and the RLC circuit. In figure 3 we showed
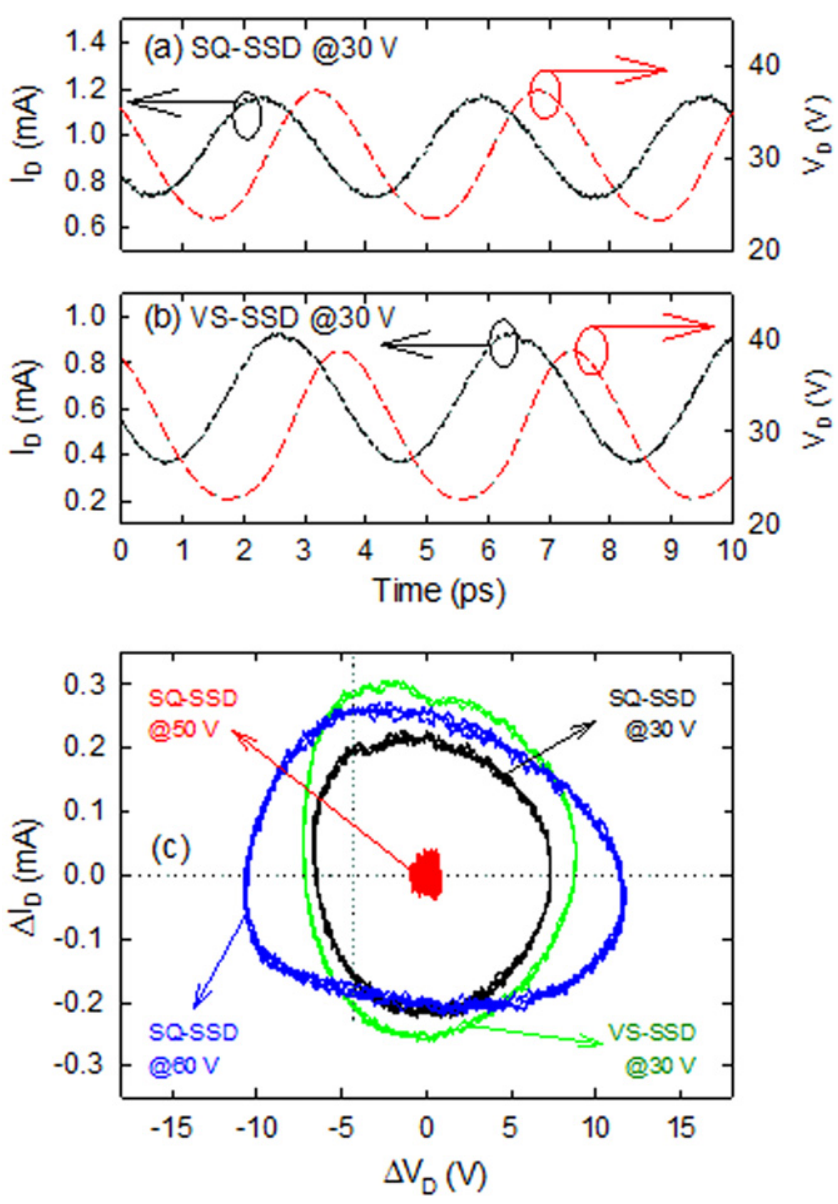

Figure 7. Time sequences of current and voltage in (a) the SQ-SSD and (b) the VS-SSD sketched in figure 2, connected in series with a resonant circuit with $\mathrm{R}=0.5 \mathrm{M} \Omega, \mathrm{C}=0.01 \mathrm{fF}$, and $\mathrm{L}=12 \mathrm{nH}$, at room temperature. The DC bias is $V_{\mathrm{DC}}=30 \mathrm{~V}$. (c) shows the phase plane portrait of $\Delta I(t)-\Delta V(t)$ for the VS-SSD at V $=30 \mathrm{~V}$ and for the SQ-SSD at $\mathrm{V}=30,50$, and $60 \mathrm{~V}$.

that our simulator reproduces satisfactorily the experimental $I-V$ curves for the SSDs when heating effects are considered. The MC results for (a) $\eta$ and (b) $f_{\mathrm{MC}}$ have been plotted in figure 6 as a function of the DC bias at room temperature and considering $R_{\mathrm{th}}=10 \times 10^{-4} \mathrm{~K} /(\mathrm{W} / \mathrm{m})$ in the simulations. Surprisingly, the efficiency obtained when heating effects are considered is higher than at $300 \mathrm{~K}$, reaching a maximum of about $0.6 \%$ for the VS-SSD (at a temperature higher than $450 \mathrm{~K})$ with similar oscillation frequencies, which are practically fixed by the external circuit. This can be attributed to the fact that the particular values of $\mathrm{R}, \mathrm{L}$, and $\mathrm{C}$ used for these MC simulations are not optimized for the devices at $300 \mathrm{~K}$, but they better match the characteristics of the SSDs when operating at high temperature (because of an increase of the device resistance). Indeed, the efficiency of the VS-SSD can be even increased to $0.8 \%$ at $V_{\mathrm{DC}}=25 \mathrm{~V}$ by increasing both C and $\mathrm{L}$ to $\mathrm{C}=0.014 \mathrm{fF}$ and $\mathrm{L}=16 \mathrm{nH}$, or to $0.72 \%$ with $\mathrm{C}=0.022 \mathrm{fF}$ and $\mathrm{L}=12 \mathrm{nH}$, using a higher capacitance that may help to compensate the inhibiting oscillation effect of the crosstalk (that will be discussed later), since for $\mathrm{N}=25$ the value of $\mathrm{C}$ is already $5.5 \mathrm{fF}$. 

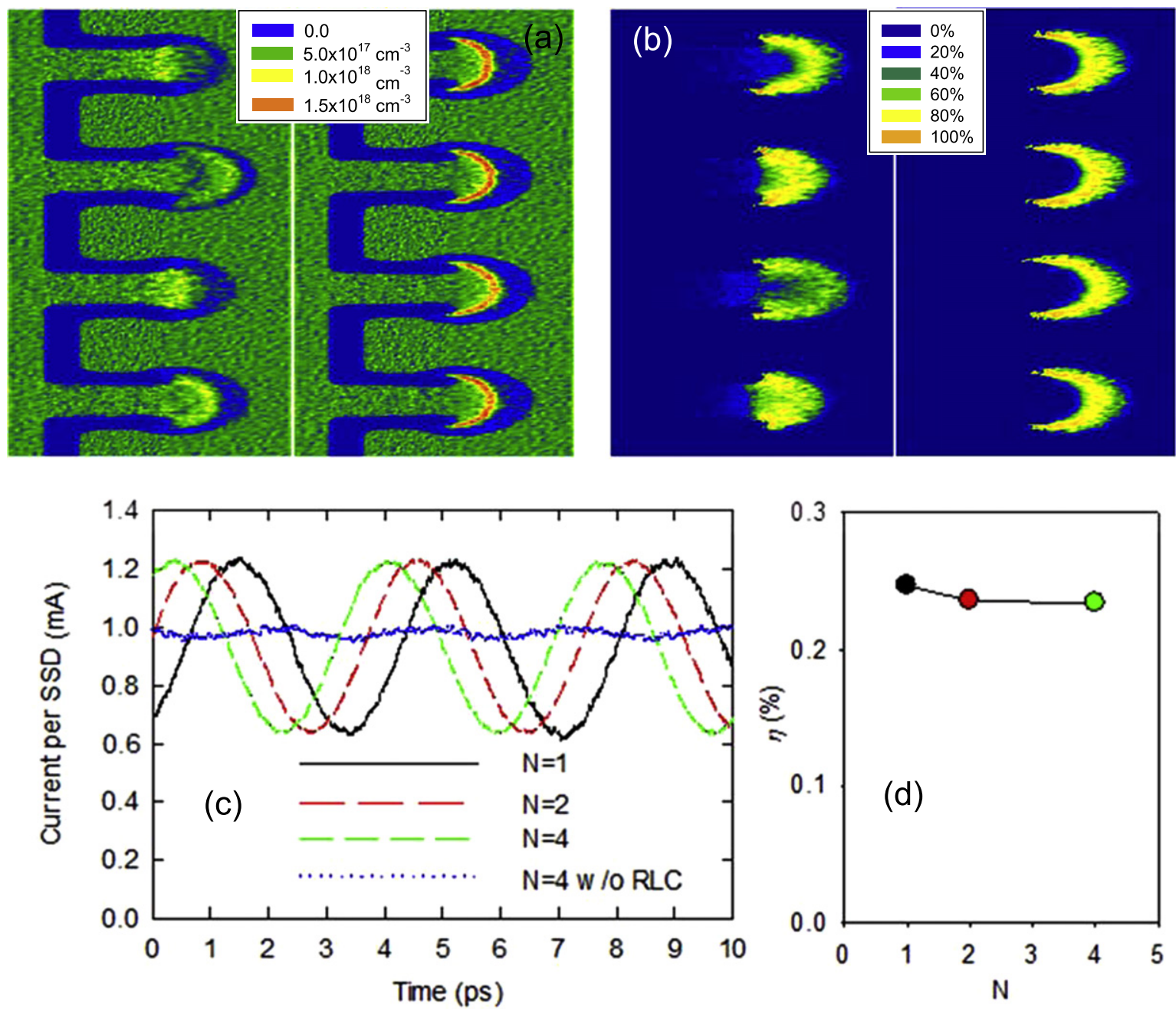

Figure 8. Contour plots of (a) electron concentration and (b) U-valley occupation obtained in the MC simulation of four SQ-SSDs in parallel with (right) and without (left) the RLC tank. (c) Time evolution of the total current per SSD in arrays of N parallel SQ-SSDs connected in series with the resonant circuit. For comparison, the result of the simulation obtained directly applying the DC voltage for the case of $\mathrm{N}=4$ is also plotted. (d) DC to AC conversion efficiency as a function of $\mathrm{N}$ when using the RLC circuit. The parameters used are $\mathrm{R}=0.5 \mathrm{~N}^{-1} \mathrm{M} \Omega$, $\mathrm{C}=0.01 \times \mathrm{N} \mathrm{fF}$, and $\mathrm{L}=12 \mathrm{~N}^{-1} \mathrm{nH}, \mathrm{T}=300 \mathrm{~K}$ and $V_{\mathrm{DC}}=30 \mathrm{~V}$.

\subsection{Parallelization and synchronization}

When several diodes are fabricated in parallel, the absence of synchronization between Gunn self-oscillations taking place in different channels could strongly deteriorate the overall signal. To illustrate this point, we have obtained snapshots of the electron concentration and the U-valley occupation from the MC simulation of four diodes in parallel without and with an RLC circuit, as shown in figures 8(a) and (b), respectively. A clear diphase between domains in the different channels is observed when the DC bias is applied directly to the array of SSDs (left side of figures 8(a) and (b)), but when adding a parallel RCL resonant circuit in series, the synchronization of the oscillations is achieved, as observed in the right side of figures 8(a) and (b), in which the domain is in the same evolution stage in the four diodes. As a result, MC simulations of arrays of two and four SSDs in parallel show that when a DC bias is applied directly in the absence of a resonant circuit, the mean current is proportional to the number of diodes, but the amplitude of the current oscillations does not scale in the same way due to pronounced desynchronization effects. Fortunately, this problem is solved when a resonant circuit is connected to the array of SSDs, as observed in figure 8(c), showing the time-sequences of the current obtained from one single diode, and two and four diodes in parallel, with an inter-distance of $800 \mathrm{~nm}$, when $\mathrm{R}=0.5 \mathrm{M} \Omega$ / $\mathrm{N}, \mathrm{C}=0.01 \mathrm{fF} \times \mathrm{N}$, and $\mathrm{L}=12 \mathrm{nH} \mathrm{N}^{-1}$ are considered for the resonant circuit. This scaling of $\mathrm{R}, \mathrm{L}$, and $\mathrm{C}$ with $\mathrm{N}$ allows for the same resonant frequency of the circuit. Figure 8(d) reports the corresponding values of $\eta$. In order to establish a proper comparison, the current per diode has been plotted in the figure (total current divided by the number of diodes $I_{\mathrm{D}} / \mathrm{N}$ ). The employment of an RLC resonant circuit in series with $\mathrm{N}$ parallel SSDs ensures the coherence between the Gunn oscillations (of about $270 \mathrm{GHz}$ ), thus providing a similar DC to AC conversion efficiency. Otherwise, in the absence of RLC resonant circuit, the current oscillations in each diode are desynchronized. Figure 8(c) also presents the time evolution of the current corresponding to $\mathrm{N}=4$ in the absence of 
Table 1. Values of the elements of the parallel RLC resonant circuit considered in the simulations for several values of the number of parallel diodes N. The dissipated DC power in the SQ- and VS-SSDs for $V_{\mathrm{DC}}$ of 25 and $20 \mathrm{~V}$, respectively, is also indicated.

\begin{tabular}{llllll}
\hline & $\mathrm{R}=0.5 \mathrm{M} \Omega N^{-1}$ & $\mathrm{~L}=12 \mathrm{nH} N^{-1}$ & $\mathrm{C}=0.01 \mathrm{fFx} N$ & $\mathrm{P}_{\mathrm{DC}}$ SQ-SSD @ 25 V & $\mathrm{P}_{\mathrm{DC}} \mathrm{VS}-\mathrm{SSD} @ 20 \mathrm{~V}$ \\
\hline $\mathrm{N}=1$ & $0.5 \mathrm{M} \Omega$ & $12 \mathrm{nH}$ & $0.01 \mathrm{fF}$ & $25 \mathrm{~mW}$ & $14 \mathrm{~mW}$ \\
$\mathrm{~N}=10$ & $50 \mathrm{~K} \Omega$ & $1.2 \mathrm{nH}$ & $0.1 \mathrm{fF}$ & $0.25 \mathrm{~W}$ & $0.14 \mathrm{~W}$ \\
$\mathrm{~N}=100$ & $5 \mathrm{~K} \Omega$ & $120 \mathrm{pH}$ & $1.0 \mathrm{fF}$ & $2.5 \mathrm{~W}$ & $1.5 \mathrm{~W}$ \\
$\mathrm{~N}=500$ & $25 \mathrm{~K} \Omega$ & $24 \mathrm{pH}$ & $5.0 \mathrm{fF}$ & $12.5 \mathrm{~W}$ & $7.5 \mathrm{~W}$ \\
\hline
\end{tabular}

an RLC resonant circuit connected in series. In this case, current oscillations with around $350 \mathrm{GHz}$ are observed, but with a significantly lower amplitude as a consequence of the desynchronization.

The values adopted in the previous simulations for the elements of the RLC resonant circuit may appear unfeasibly low for a classical circuit approach. However, as described before, the values to be used when considering an array of $\mathrm{N}$ channels in parallel instead of a single SSD must be scaled accordingly. For $\mathrm{N}$ parallel diodes, the current obtained is the one provided by a single SSD multiplied by a factor $\mathrm{N}$ (a rule that also follows the capacitance of the diode). Therefore, if $\mathrm{R} / \mathrm{N}, \mathrm{L} / \mathrm{N}$, and $\mathrm{C} \times \mathrm{N}$ are taken as the values of the resonant circuit of the array of $\mathrm{N}$ channels, they provide the same efficiency obtained for $\mathrm{N}=1$, as shown in figure $8(\mathrm{~d})$. Table 1 shows the values of $\mathrm{R}, \mathrm{L}$, and $\mathrm{C}$ that provide the same efficiency $(0.18 \%)$ as a single $\mathrm{SQ}-\mathrm{SSD}$ for $\mathrm{R}=0.5 \mathrm{M} \Omega$, $\mathrm{C}=0.01 \mathrm{fF}$, and $\mathrm{L}=12 \mathrm{nH}$ for different numbers of parallel diodes N. In order to obtain Gunn oscillations with few parallel SSDs it is necessary to use very small values of C. This may be a problem for the experimental achievement of current oscillations, since they can be of the order of the crosstalk capacitances $\mathrm{C}_{\text {talk }}$ that could block the appearance of the Gunn oscillations in the fabricated devices. MC simulations including the crosstalk capacitances (see figure 1), experimentally estimated to be about $5 \mathrm{fF}$ (regardless of the value of $\mathrm{N}$, since they result mainly from the geometry of the access regions) and show that oscillations are still generated by RLC circuits as long as a value of $\mathrm{C}$ higher than $5 \mathrm{fF}$ is used, but with a slightly lower efficiency. In fact, this parasitic capacitance can be at the origin of the absence of experimental evidence of Gunn oscillations in the experimental devices [8]. This problem can be overcome by using a number of parallel SSDs that fulfill the condition $\mathrm{C}>\mathrm{C}_{\text {talk }}$, as happens for $\mathrm{N}>500$. However, in such a case, the thermal management may be problematic, since already 100 SSDs would need a DC power above the $1 \mathrm{~W}$ level to operate (even if the $\mathrm{V}$-shape geometry is able to decrease it almost to the half). Technologically, the crosstalk capacitances can be decreased by improving the design of the accesses and moving the ohmic contacts farther away, so that the number of channels to have $\mathrm{C}>\mathrm{C}_{\text {talk }}$ can be reduced as much as possible. Indeed, we expect to improve our mask design in future fabrication runs, aiming to achieve $\mathrm{C}_{\mathrm{talk}}<1.0 \mathrm{fF}$ so that the number of parallel SSDs can be reduced below $\mathrm{N}=100$. Furthermore, other circuit configurations of the resonator using higher capacitances may be used in order to reduce the influence of the crosstalk capacitance.

\section{Conclusions}

A semiclassical ensemble MC simulator self-consistently coupled with a 2D Poisson solver has been employed to analyzed the Gunn-oscillation operation of GaN-based SSDs, working in series with a resonant RLC parallel circuit, in terms of the DC to AC conversion efficiency and the currentoscillation frequency. The simulator, which allows us to take into account the heating effects by using a self-consistent temperature model, has been further validated by the good agreement with the $I-V$ curves measured in fabricated SSDs.

According to our MC results, an efficiency of at least $0.18 \%$ can be achieved when including a proper RLC resonant circuit, with the possibility of frequency tunability from $270-350 \mathrm{GHz}$ at room temperature. VS-SSDs have been found to be more efficient than SQ-SSDs, providing an $\eta$ value of at least $0.35 \%$. This value can be increased up to at least $0.80 \%$, even when considering the degradation of the Gunn oscillations produced by self-heating effects.

The values of the elements of the RLC resonant circuit producing optimum oscillations can be modified if considering, instead of a single SSD, an array of channels in parallel (as commonly fabricated, given the enhancement of the current and the eventual emitted power). $\mathrm{R} / \mathrm{N}, \mathrm{L} / \mathrm{N}$, and $\mathrm{C} \times \mathrm{N}$ are the values of the resonant circuit elements that must be used with an array of $\mathrm{N}$ channels if one wants to obtain the same efficiency as for one single diode. This scaling may help to overcome the negative effect of the crosstalk capacitance of the diodes. Furthermore, a perfect synchronization of the Gunn oscillations of every single SSD in parallel is achieved by the connection of the SSDs' array to the resonant circuit.

\section{Acknowledgments}

This work has been partially supported by the European Commission through the ROOTHz Project ICT-2009243845, the Dirección General de Investigación (MICINN) through Project TEC2010-15413, and by the Consejería de Educación, Junta de Castilla y León through Project SA052U13. This work has benefited from the facilities of the ExCELSiOR-Nanoscience Characterization Center. 


\section{References}

[1] Miles R E, Zhang X-C, Eisele H and Krotkus A (ed) 2007 Terahertz Frequency Detection and Identification of Materials and Objects (New York: Springer)

[2] Song M, Missous M, Omling P, Peaker A R, Samuelson L and Seifert W 2003 Unidirectional electron flow in a nanometerscale semiconductor channel: a self-switching device Appl. Phys. Lett. 83 1881-3

[3] Xu K Y, Wang G and Song A M 2008 Gunn oscillations in a self-switching nanodiode Appl. Phys. Lett. 93233506

[4] Mateos J, Vasallo B G, Pardo D and González T 2005 Operation of high-frequency performance of nanoscale unipolar rectifying diode Appl. Phys. Lett. 86212103

[5] Balocco C, Kasjoo R S, Lu X F, Zahng L Q, Alimi Y, Winnert S and Song A M 2011 Room-temperature operation of a unipolar nanodiode at terahertz frequencies Appl. Phys. Lett. 98223501

[6] Sangaré P, Ducournau G, Grimbert B, Brandli V, Gaquière C, Iñiguez-de-la-Torre A, Iñiguez-de-la-Torre I, Mateos J and González T 2013 Experimental demonstration of direct terahertz detection at room-temperature in $\mathrm{AlGaAs} / \mathrm{GaN}$ asymmetric nanochannels J. Appl. Phys. 113034305

[7] Westlund A, Sangaré P, Ducournau G, Nilsson P, Gaquière C, Desplanque L, Wallart X and Grahn J 2011 Terahertz detection in zero-bias InAs self-switching diodes at room temperature Appl. Phys. Lett. 103133504

[8] Iñiguez-de-la-Torre A, Iñiguez-de-la-Torre I, Mateos J, González T, Sangaré P, Gaucher M, Grimbert B, Brandli V, Ducournau G and Gaquière C 2012 Searching for THz Gunn oscillations in GaN planar nanodiodes J. Appl. Phys. 111 113705

[9] Yang L, Long S, Guo X and Hao Y 2012 A comparative investigation on sub-micrometer $\mathrm{InN}$ and $\mathrm{GaN}$ gunn diodes working at terahertz frequency J. Appl. Phys. 111104514

[10] Macpherson R F, Dunn G M and Pilgrim N J 2008 Simulation of gallium nitride Gunn diodes at various doping levels and temperatures for frequencies up to $300 \mathrm{GHz}$ by Monte Carlo simulation, and incorporating the effects of thermal heating Semicond. Sci. Technol. 23055005

[11] Sevik C and Bulutay C 2004 Gunn oscillations in GaN channels Semicond. Sci. Technol. 19 S188

[12] Yilmazoglu O, Mutamba K, Pavlidis D and Karaduman T 2008 First observation of bias oscillations in GaN Gunn diodes on GaN substrate IEEE Trans. Elec. Dev. 551563

[13] Alekseev E and Pavlidis D 2000 Large-signal microwave performance of GaN-based NDR diode oscillators SolidState Electron. 44941

[14] Ma N et al 2010 Current-controlled negative differential resistance effect induced by Gunn-type instability in n-type GaN epilayers Appl. Phys. Lett. 96242104

[15] Jacoboni C and Lugli P 1989 The Monte Carlo method for semiconductor device simulation (New York: Springer)

[16] Millithaler J-F, Iñiguez-de-la-Torre I, Iñiguez-de-la-Torre A, González T, Sangaré P, Ducournau G, Gaquière C and Mateos J 2014 Optimized V-shape design of GaN nanodiodes for the generation of Gunn oscillations Appl. Phys. Lett. 104073509
[17] Shiktorov P, Starikov E, Gruzinskis V, Pérez S, González T, Reggiani L, Varani L and Vaissière J C 2006 Theoretical investigation of Schottky-barrier diode noise performance in external resonant circuits Semicond. Sci. Technol. 21550

[18] Iñiguez-de-la-Torre I, Mateos J, Pardo D and González T 2009 Noise and terahertz rectification linked by geometry in planar asymmetric nanodiodes Appl. Phys. Lett. 94093512

[19] García S, Pérez S, Iñiguez-de-la-Torre I, Mateos J and González T 2014 Comparative Monte Carlo analysis on InPand GaN-based Gunn diodes J. Appl. Phys. 115044510

[20] Xu K Y, Lu X F, Song A M and Wang G 2008 Terahertz harmonic generation using planar nanoscale unipolar diode at zero bias Appl. Phys. Lett. 92163503

[21] Iñiguez-de-la-Torre I, Mateos J, González T, Pardo D, Galloo J S, Bollaert S, Roelens Y and Cappy A 2007 Influence of the surface charge on the operation of ballistic T-branch junctions: a self-consistent model for Monte Carlo simulations Semicond. Sci. Technol. 22663

[22] Mateos J, Vasallo B G, Pardo D, González T, Galloo J S, Roelens Y, Bollaert S and Cappy A 2003 Ballistic nanodevices for terahertz data processing: Monte Carlo simulations Nanotechnology 14117

[23] Mateos J, Vasallo B G, Pardo D, González T, Galloo J S, Bollaert S, Roelens Y and Cappy A 2003 Microscopic modeling of nonlinear transport in ballistic nanodevices IEEE Trans. Electron Devices 501897

[24] Millithaler J F, Íñiguez-de-la-Torre I, González T, Mateos J, Sangaré P, Ducournau G and Gaquière C 2013 Monte Carlo analysis of thermal effects in self-switching diodes Spanish Conf. on Electron Devices 45

[25] Prejs A, Wood S, Pengelly R and Pribble W 2009 Thermal analysis and its application to high power GaN HEMT amplifiers IEEE MTT-S Int. Microwave Symp. Digest 2009 917-20

[26] Asgari A, Kalafi M and Faraone L 2005 A quasi-twodimensional charge transport model of AlGaN/GaN high electron mobility transistors (HEMTs) Physica E 28491

[27] Kim H, Min H S, Tang T W and Park Y J 1991 An extended proof of the Ramo-Shockley theorem Solid-State Electron. 341251

[28] García S, Vasallo B G, Mateos J and González T 2013 Timedomain Monte Carlo simulations of resonant-circuit operation of GaN Gunn diodes Spanish Conf. on Electron Devices 79

[29] Dunn G M, Phillips A and Topham P J 2001 Current instability in power HEMTs Semicond. Sci. Technol. 16562

[30] Gruzinskis V, Starikov E, Shiktorov P, Reggiani L and Varani L 1994 Linear and nonlinear analysis of microwave power generation in submicrometer $\mathrm{n}^{+} \mathrm{nn}^{+} \mathrm{InP}$ diodes J. Appl. Phys. 765260

[31] Barry E A, Sokolov V N, Kim K W and Trew R J 2010 Largesignal analysis of terahertz generation in submicrometer GaN diodes IEEE Sensor Journal 10765

[32] Eisele H and Haddad G I 1998 Efficient power combining with D-band (110-170 GHz) InP Gunn devices in fundamentalmode operation IEEE Microwave and Guided Wave Lett. 824

[33] Eisele H 2007 Dual Gunn device oscillator with $10 \mathrm{~mW}$ at $280 \mathrm{GHz}$ Electron. Lett. 43636 